\title{
The application of LM-BP Neural Network in the Circulating Fluidized Bed Unit
}

\author{
Mengjie $\mathrm{Hu}^{1, a}$, Hujun Ling ${ }^{2, b}$ and Dongxu Liü ${ }^{2, \mathrm{c}}$ \\ ${ }^{1}$ Inner Mongolia University of Technology, College of electric power, Huhhot 010080, China; \\ ${ }^{2}$ Inner Mongolia University of Technology, Huhhot 010080, China \\ a359010375@qq.com, b442682716@qq.com
}

\section{Keywords: LM-BP Neural Network, Circulating Fluidized Bed Unit}

\begin{abstract}
Circulating fluidized bed technology is one of the developing direction of clean coal technology which is more reality, and one of the effective ways to solve the thermal power to meet the increasingly stringent environmental requirements. Due to the dynamic process of circulating fluidized bed boiler with control of multivariable, nonlinearity, large delay, strong coupling, etc., the general control strategy can not meet the actual requirements. This paper presents a generalized predictive control strategy based on LM-BP neural network, and validates the feasibility and superiority of control strategy through the comparison with traditional PID control strategy.
\end{abstract}

\section{Introduction}

Today's severe energy problem and environmental problems forced the power generation industry focus on looking for clean energy and related technology. Circulating Fluidized Bed(Circulating Fluidized Bed, CFB) combustion technology was developed in the $1980 \mathrm{~s}$, the CFB combustion technology has been rapid development, as a kind of clean coal combustion technology, is now the CFB coal-fired energy problem solving and one of the effective methods of environmental problems.

Compared with the conventional pulverized coal fired Boiler, CFBB (Circulating Fluidized Bed Boiler, CFBB) has obvious differences in structure and combustion mechanism. In the control system, there are also many different places between the CFBB and conventional pulverized coal furnace, with one of the most important is the furnace bed temperature control of the combustion chamber.

Bed temperature is the key to the CFBB can keep stable operation. In the process of normal combustion boiler operation, the bed temperature must be maintained within the prescribed scope, bed temperature control is the precondition of other adjustment and control. In practical applications, the CFBB control system design is mainly adopt the traditional PID control, the control method can't meet the requirements of the ideal control effect. So, study the application of advanced control technology in the CFBB control has important practical value.

\section{LM-BP Neural Network}

\subsection{BP Neural Network.}

BP (Back Propagation) network, namely the error Back Propagation, is a kind of artificial neural network. BP network is a one-way transmission of multi-layer forward networks. The structure of BP network as shown in figure 2-1. BP network has not only the input layer nodes, the output layer nodes, and there is a layer or layers of hidden nodes, each neuron in a node, the network node in the one tree without any connection, layer after layer to node through the right connection.

\subsection{LM-BP Neural Network.}

Due to the traditional BP algorithm's slow convergence speed and is easily convergence to the local minimum, so the effective improvement of $\mathrm{BP}$ algorithm became thehot spot. LM (Levenberg Marquardt, LM) algorithm is one of the optimization algorithms, is the most widely used nonlinear least squares algorithm. LM algorithm using the approximation of the second derivative information, has second order convergence speed, which is faster than gradient method. LM algorithm both local 
convergence properties of Newton method, and the gradient descent algorithm of global features. It's a fusion of Newton's method and gradient descent method.

In the LM algorithm, set $x$ as the input samples of neural network, and neural network error index function for :

$$
E(x)=\frac{1}{2} \sum_{i=1}^{N}\left\|Y_{i}-\hat{Y}_{i}\right\|^{2}=\frac{1}{2} \sum_{i=1}^{N} e_{i}^{2}(x)
$$

In the type, $Y_{i}$ for the desired output vector of the neural network, $\hat{Y}_{i}$ for the actual output vector of the neural network, $e_{i}(x)$ for the error.

Set $e(x)=\left[e_{1}(x), e_{2}(x), \cdots e_{N}(x)\right]^{T}, w(k)$ for the weight vector of the Kth iteration, $\mathrm{M}$ for the dimension. The new weight vector $w(k+1)$ can be obtained by the following rules:

$$
\begin{gathered}
w(k+1)=w(k)+\Delta w(k)=w(k)-H_{k}^{-1} g_{k} \\
H_{k}=\left.\nabla^{2} E(x)\right|_{x=w(k)}=J^{T}(x) J(x)+S(x) \\
g_{k}=\left.\nabla E(x)\right|_{x=w(k)}=J^{T}(x) e(x)
\end{gathered}
$$

Among them, $J$ is the Jacobian matrix.

$$
\begin{gathered}
J=\left[\begin{array}{cccc}
\frac{\partial e_{1}}{\partial w_{1}} & \frac{\partial e_{1}}{\partial w_{2}} & \cdots & \frac{\partial e_{1}}{\partial w_{n}} \\
\frac{\partial e_{2}}{\partial w_{1}} & \frac{\partial e_{2}}{\partial w_{2}} & \cdots & \frac{\partial e_{2}}{\partial w_{n}} \\
\vdots & \vdots & \ddots & \vdots \\
\frac{\partial e_{N}}{\partial w_{1}} & \frac{\partial e_{N}}{\partial w_{2}} & \cdots & \frac{\partial e_{N}}{\partial w_{n}}
\end{array}\right] \\
S(x)=\sum_{i=1}^{N} e_{i}(x) \nabla^{2} e_{i}(x)
\end{gathered}
$$

Set the $H: H=J^{T}(x) J(x)+\mu I$

Among them, $\mu>0$ for the proportion coefficient is usually very small, $I$ is the unit matrix.

Eventually get a weight correction method:

$$
\Delta w=-\left[J^{T}(x) J(x)+\mu I\right]^{-1} J^{T}(x) e(x)
$$

Train the LM-BP network and the traditional BP network with the CFBB model, training results are shown in figure 4-1 and figure 4-2. It can be seen that LM-BP network improves the convergence speed obviously.

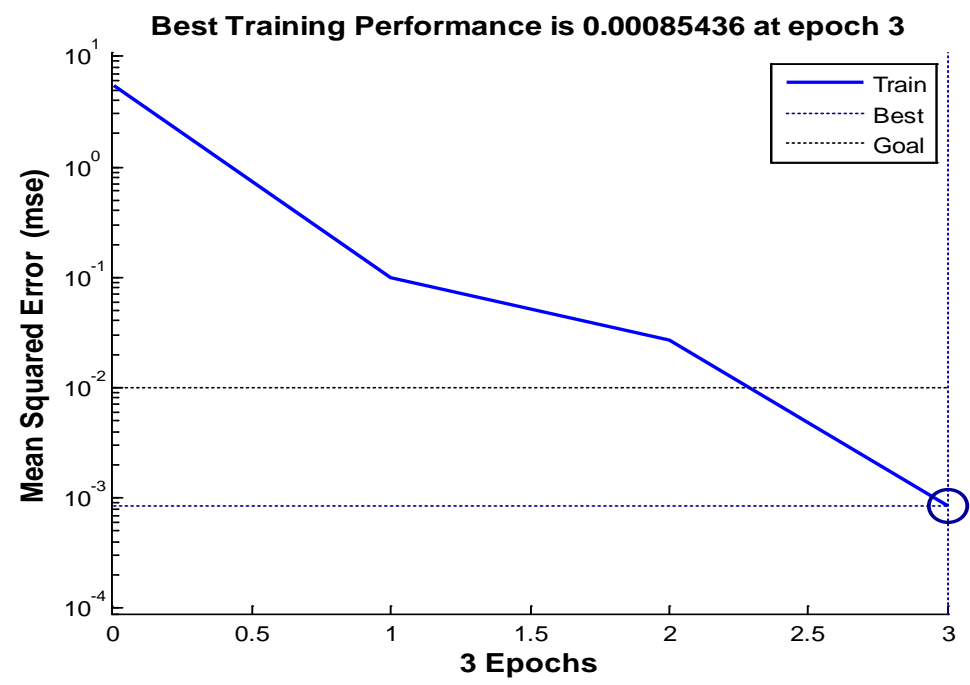

Fig.4-1 LM-BP neural network trained error curve 


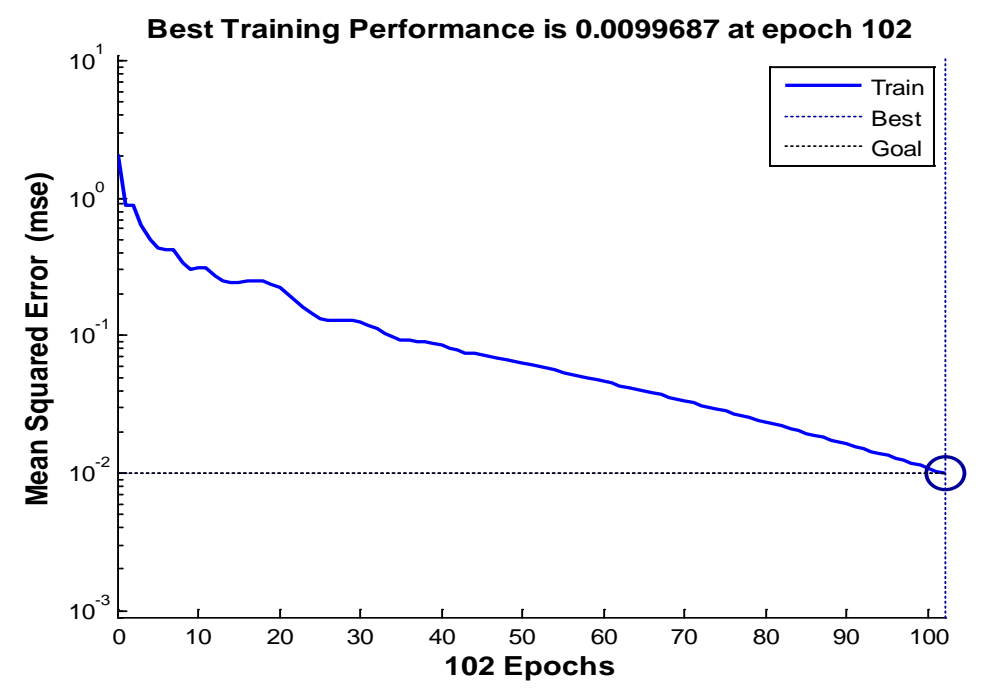

Fig.4-2 BP neural network trained error curve

\section{The application of LM-BP in the CFB}

To verify the robustness of the LM-BP coordinated control on the CFBB, this article combine the LM-BP network and generalized predictive control strategy, and do the simulation in view of the tracking ability and the control of bed temperature and the main steam pressure of the CFBB with the circumstance of large range fluctuation of the load.

The output curve of the CFBB in LM-BP generalized predictive control under the variable load control is shown in figure 3-1. The curve of the CFBB in PID control is shown in figure 3-2.
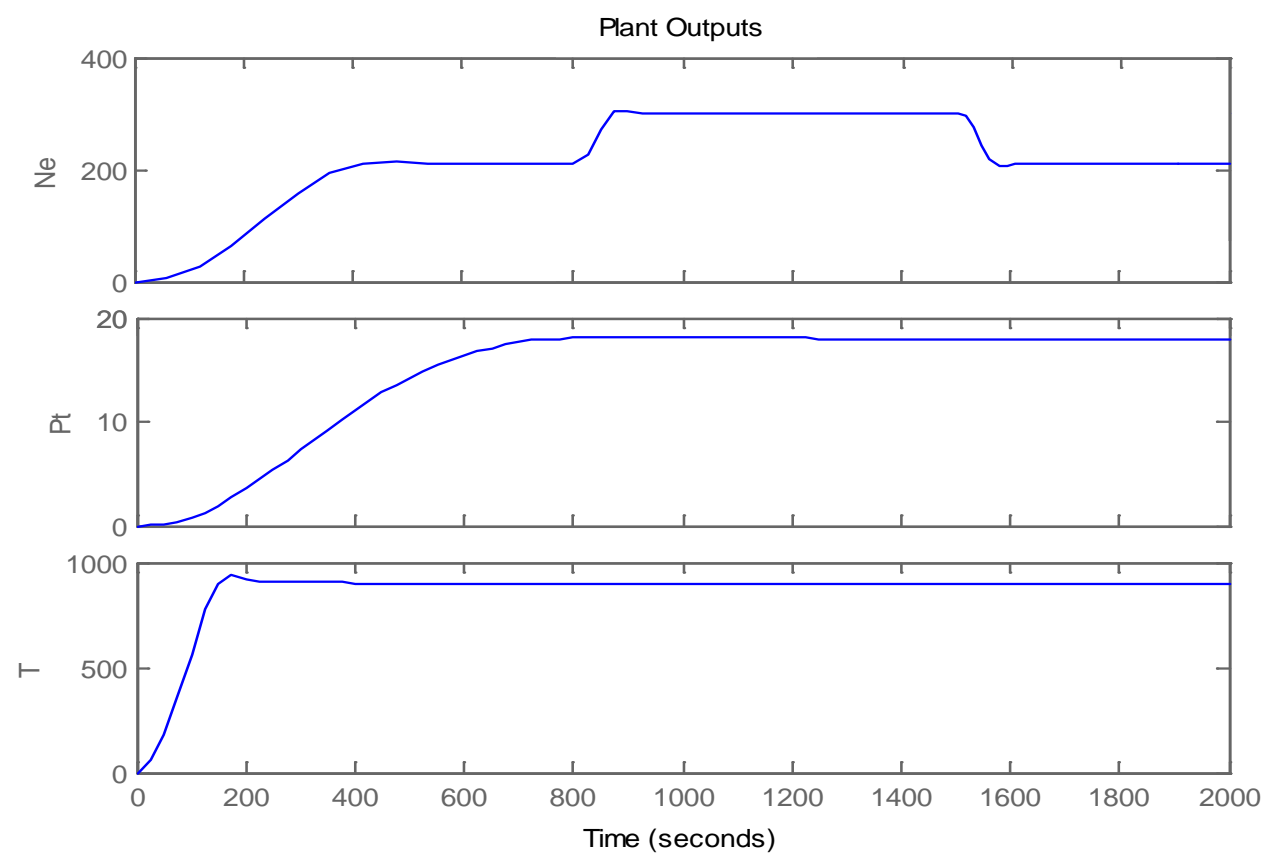

Fig.3-1 $\mathrm{N}_{\mathrm{e}}$ and $\mathrm{P}_{\mathrm{t}}$ response curve of changing load control based on LM-BP GPC 

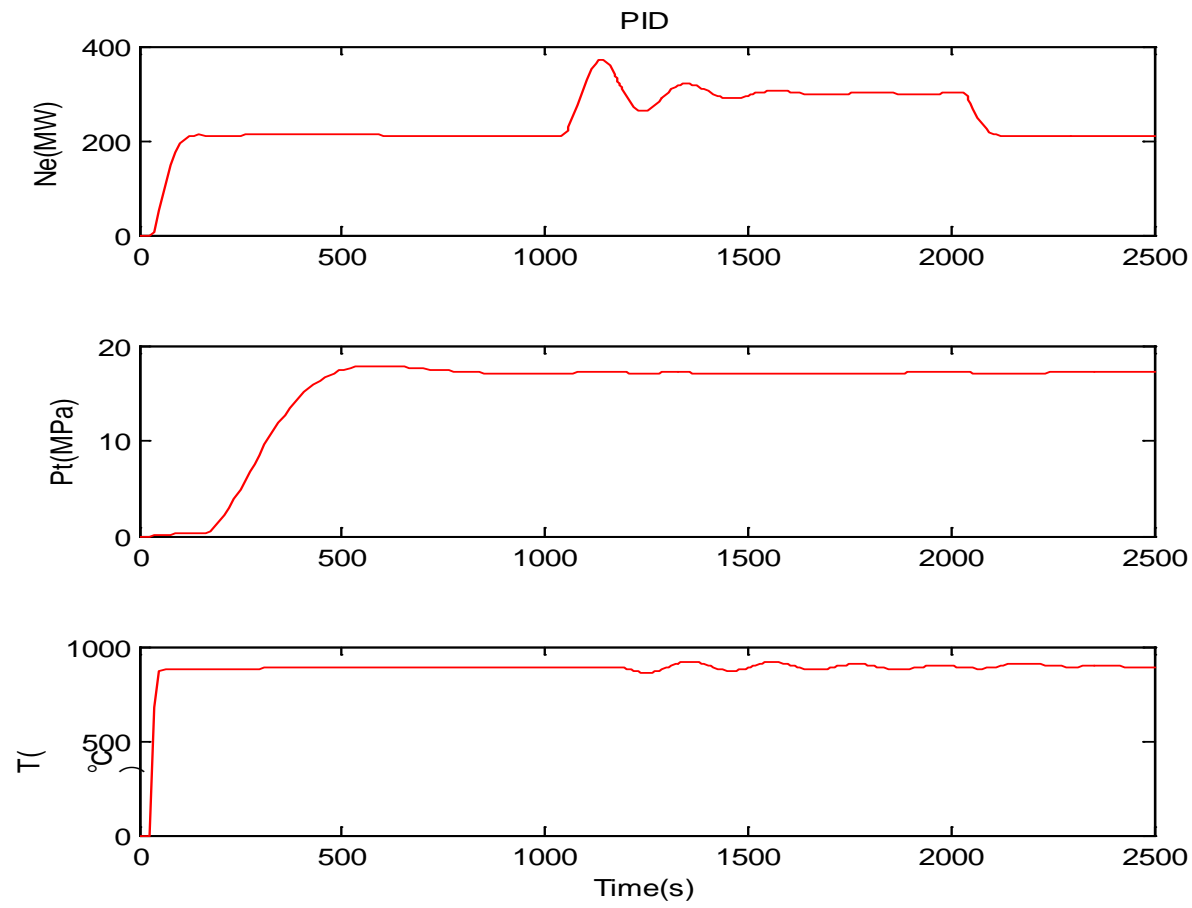

Fig.3-2 response curve of changing load control based on PID

It can be seen by the simulation results that the conventional PID controller has good dynamic performance and stable performance under $70 \%$ load conditions. When the load roses from $70 \%$ to $100 \%, N_{e}$ has large overshoot, which cannot meet the practical requirements of load control, and the regulation time of $N_{e}$ is longer than LM-BP generalized predictive control.

\section{Summary}

To sum up, LM-BP generalized predictive control has good control effect on the coupling system which is multivariable and with large lag. The controller can guarantee the stability of the system under the condition of invariable parameters, especially when there is a change in the system model. There will not appear large overshoot in the process of adjusting, which satisfies the requirement of practical production well.

\section{References}

[1]. Yue, G.X., Yang, H.R., Lu, J.F.,Zhang, H. Latest development of CFB boilers in China[C]. 20 ${ }^{\text {th }}$ International Conference on Fluidized Bed Combustion, Xian, China, 2009. Unavailable, United Kingdom,2009:3-12.

[2]. L. Zhao, F. Qian. Tuning the structure and parameters of a neural network using cooperative binary-real particle swarm optimization [J]. Expert Systems with Applications, 2011, 38(5): 4972-4977.

[3]. Huseyin Demircioglu,Erean Karasu. Generalized Predictive control: a Practical application andComparison of discrete and continuous-time versions[J].IEEE control system magazine,2000,20(5):36-47.

[4]. Demircioglu H, Yavuzyilmaz C. Constrained Predictive control in continuous-time [J].IEEEControl Systems Magazine.2002,57-67. 